\title{
Coriolis induced compressibility effects in rotating shear layers
}

\author{
Bernard J. Geurts ${ }^{1,2}$, Darryl D. Holm ${ }^{3,4}$, and Arkadiusz K. Kuczaj ${ }^{1}$ \\ 1 Multiscale Modeling and Simulation, NACM, J.M. Burgers Center, Faculty \\ EEMCS, University of Twente, P.O. Box 217, 7500 AE Enschede, the \\ Netherlands: b.j.geurts@utwente.nl \\ 2 Anisotropic Turbulence, Fluid Dynamics Laboratory, Department of Applied \\ Physics, Eindhoven University of Technology, P.O. Box 513, 5300 MB \\ Eindhoven, the Netherlands \\ 3 Mathematics Department, Imperial College London, SW7 2AZ, London, UK \\ 4 CCS2, Los Alamos National Laboratory, Los Alamos, NM 87545, USA
}

Rotation about a fixed axis introduces a competition between two- and threedimensional tendencies in a turbulent flow $[1,2,3]$. At strong rotation rates, this competition expresses itself, e.g., by suppressing fluid motion along the axis of rotation. This yields a reduced mixing-efficiency in a rotating frame of reference that is particularly relevant in the context of atmospheric and oceanic flows. The exchange of gases between atmosphere and oceans, the transport of heat and the spreading of pollutants or large-scale planktonpopulations, are all significantly affected by rotation in large-scale environmental flows.

The canonical problem of flow in a horizontal temporal mixing layer, subjected to rotation about a vertical axis is investigated with the use of direct numerical simulation. The compressible mixing layer is considered, composed of two counter-flowing horizontal slabs of fluid [4]. Periodic boundary conditions are adopted in the horizontal directions and free-slip conditions apply in the vertical far-field. High order finite volume discretization and explicit, compact storage, four-stage Runge-Kutta time-stepping are used.

Qualitatively, in the non-rotating case the flow shows vigorous mixing in the vertical direction leading to a complex three-dimensional flow. A snapshot of the vertical vorticity component in the turbulent regime is shown in Fig. 1(a). Adding rotation to this flow may completely alter the transitional and developed flow. As a result of rotation, the flow breaks up into vertically oriented cells that collectively undergo an oscillatory motion (cf. Fig. 1(b)). The frequency of this oscillation increases with rotation rate.

The effect of rotation was investigated at a Reynolds number $R e=50$, based on the initial momentum-thickness of the shear layer, and a low convective Mach number of $M=0.2$. The strength of the rotation is characterized 

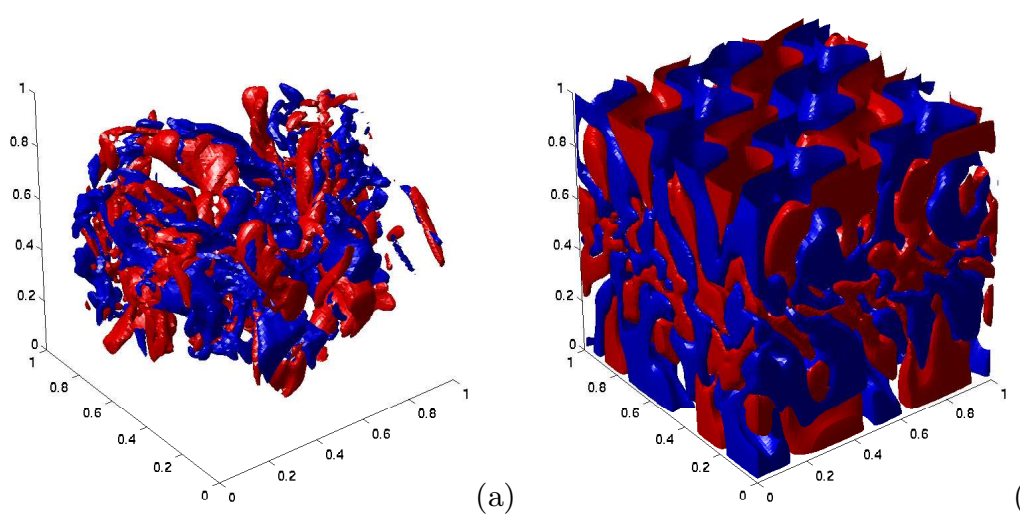

(b)

Fig. 1. Snapshot of the vertical component of vorticity in the developed regime at $t=90$ for (a) the non-rotating case $R o=\infty$ and (b) rotating flow at $R o=10$. Positive and negative values are shown in different colors.

by the Rossby number $R o=u_{r} /\left(2 \Omega_{r} \ell_{r}\right)$ where $u_{r}, \Omega_{r}$ and $\ell_{r}$ are reference velocity, rotation-rate and length-scale used in the non-dimensionalization. At high Rossby numbers Ro, i.e., low rotation rates, the flow develops very similarly to incompressible flow. However, at sufficiently low Rossby numbers $R o<1$, rotation was found to induce significant compressibility effects. These give rise to rapidly varying small scale flow-features. By comparing results obtained at a variety of time-steps, we verified that the explicit Runge-Kutta time-stepping properly captures these rapid variations. The spatial resolution was found to be adequate at $256^{3}$ grid-cells for the purpose of studying the decay of the kinetic energy. Results at $256^{3}$ grid-cells were compared with lower resolutions at $192^{3}$ and $128^{3}$, confirming the accuracy.

Rotation has an indirect influence on the decay-rate of kinetic energy $E=\langle\mathbf{u} \cdot \mathbf{u}\rangle / 2$. Here $\langle\cdot\rangle$ denotes averaging over the flow-domain. The evolution of the kinetic energy may be written as

$$
\frac{d E}{d t}=D-W \quad ; \quad D=\langle p \nabla \cdot \mathbf{u}\rangle \quad ; \quad W=\langle\mathbf{S}: \mathbf{S} /(2 R e)\rangle
$$

where $p$ denotes pressure, $\mathbf{u}$ the velocity field, $\mathbf{S}$ the rate of strain tensor and $R e$ the Reynolds number. The viscous dissipation is represented in $W$ while the compressible pressure/velocity-divergence is given by $D$. In (1) there is no direct effect of rotation. Rather, rotation effects may be distinguished in the dynamics of $D$ and $W$. By investigating the various contributions to $d D / d t$ and $d W / d t$ we may identify the basic mechanisms that govern the kinetic energy dynamics, particularly the role of the Coriolis forces. The dominant mechanisms at various $R o$ in the dynamics of the viscous dissipation and the pressure/velocity-divergence can be extracted from the DNS-data. 



Fig. 2. Decay of kinetic energy at low (a) and high (b) rotation rates. In (a) $R o=\infty$ (solid), $R o=10$ (dashed), $R o=5$ (dash-dotted); in (b) $R o=1$ (solid), $R o=0.5$ (dashed) and $R o=0.2$ (dash-dotted).

The marked differences in the decay of kinetic energy in slowly and in rapidly rotating shear layers are shown in Fig. 2. At high $R o$ an increase in the rotation-rate yields a decrease in the decay rate (cf. Fig. 2(a)). This reduction in decay-rate and the strict monotonicity of $d E / d t$ are also found in incompressible flow. For sufficiently rapid rotation (in this flow $R o \lesssim 1$; Fig. 2(b)) explicit compressibility effects become dominant. The behavior of the decay-rate is completely reversed and an increase in rotation-rate yields a strong increase in the 'average decay-rate'. Moreover, the kinetic energy is no longer a monotonously decaying function of time. Next to the strictly dissipative viscous contributions, the pressure/velocity-divergence correlations are found to become dynamically significant at low $R o$. The observed rapid fluctuations are a purely compressible effect. A complete analysis is topic of current investigations.

A.K.K. gratefully acknowledges financial support from the Dutch Foundation for Fundamental Research of Matter (FOM) and from the Turbulence Working Group at Los Alamos National Laboratory - USA.

\section{References}

1. Mansour, NN, Cambon, C, Speziale, CG (1992) in Studies in Turbulence (ed. Gatski, TB, Sarkar, S, Speziale, CG). Springer.

2. Hossain, M (1994) Phys. Fluids 6:1077.

3. Smith, LM, Waleffe, F (1999) Phys. Fluids. 6:1608.

4. Geurts, BJ, Holm, DD (2006) J. of Turbulence. 71 - 33. 